\title{
3D Simulation and Analysis of AlGaN/GaN Ultraviolet Light Emitting Diodes
}

\author{
Joachim Piprek, ${ }^{\text {i }}$ Tom Katona, Steven P. DenBaars, and Simon Li ${ }^{*}$ \\ Solid-State Lighting and Display Center, Materials Department \\ University of California, Santa Barbara, CA 93106, U.S.A. \\ * Crosslight Software, Burnaby, Canada
}

\begin{abstract}
Compact ultraviolet light sources are currently of high interest for applications in solid-state lighting, short-range communication, and bio-chemical detection. Our nitride-based light-emitting diode (LED) includes AlGaN quantum wells with an emission wavelength of approximately $340 \mathrm{~nm}$. In this paper, we analyze internal device physics by three-dimensional (3D) numerical simulation. The simulation incorporated a 3D drift-diffusion model for the carrier transport, the quantum well $(\mathrm{QW})$ energy band-structure including interface polarization charges, the local $\mathrm{QW}$ spontaneous emission spectrum, as well as 3D raytracing for photon extraction. The simulation results showed good agreement with measurements. Internal physical mechanisms such as current crowding, carrier leakage, and carrier recombination were investigated. Nanoscale effects exhibited a strong influence on the LED performance.
\end{abstract}

Keywords: Ultraviolet light source, light-emitting diode, $\mathrm{LED}, \mathrm{AlGaN} / \mathrm{GaN}$ quantum wells, numerical simulation

\section{INTRODUCTION}

Compact ultraviolet (UV) light sources are currently of great interest for applications in solid-state lighting, shortrange communication, and bio-chemical detection. Prime candidates are III-Nitride-based light-emitting diodes (LEDs) with AlGaN quantum wells. Despite the interest in this technology, output powers and quantum efficiency for these devices is still relatively low compared to their visible InGaN-based counterparts. It is believed that the low efficiency results from a combination of lower ionization efficiency for both p-type and n-type dopants with added Al composition in $\mathrm{AlGaN}$, higher impurity incorporation with added $\mathrm{Al}$, and lower structural material quality of the device epitaxial layers. Detailed simulations of UV LEDs have not been previously reported to investigate the effect of fundamental physical effects on the performance limits of these devices.

For the first time, we present a three-dimensional (3D) physics-based simulation of such LEDs aimed at a better understanding of internal device physics and an improved ability to optimize the device design. We previously used 1D simulations to investigate the design of the multi-quantum well (MQW) active region. ${ }^{1}$ An improved design of the active region was developed which resulted in $80 \%$ higher output power. However, the power was still below the requirement for practical applications. We have investigated these most recent devices by advanced 3D simulation to identify performance limiting internal mechanisms. Good agreement with measurements was achieved by refinement of the physical model and by calibration of material parameters within the range reported in the literature. Based on this agreement, we were able to analyze the practical impact of micro- and nanoscale physical effects such as current crowding, carrier leakage, and built-in polarization. Device structure and experimental results are described in Section 2, followed by an outline of the theoretical model in Section 3. Section 4 discusses key material properties. Section 5 presents the primary simulation results and a discussion of their significance.

${ }^{\mathrm{i}}$ Corresponding author, e-mail: piprek@ieee.org 


\section{DEVICE STRUCTURE AND EXPERIMENTAL RESULTS}

The growth was performed in a vertical close-spaced MOCVD reactor at 760, 300, and 76 torr for the GaN, AlGaN, and AlN layers, respectively. The growth temperatures ranged from $\sim 1000-1060{ }^{\circ} \mathrm{C}$ for all layers except the AlN interlayer which was grown at $\sim 700{ }^{\circ} \mathrm{C}$. The quantum wells had a high composition $\mathrm{Al}_{0.30} \mathrm{Ga}_{0.70} \mathrm{~N}$ electron blocking layer on the p-side in an attempt to prevent carrier overflow out of the active region. No hole blocking layer was used because of the low hole mobility in these materials. A short period alloy superlattice (SPASL) was used in both the $n$ and p-type cladding to reduce the vertical resistivity. After growth, the samples were annealed at $750{ }^{\circ} \mathrm{C}$ for 15 minutes and a semi-transparent $\mathrm{Pd} / \mathrm{Au}(30 \AA / 50 \AA)$ p-contact was deposited. The mesa was formed by reactive ion etching in $\mathrm{Cl}_{2}$ gas at $200 \mathrm{~W}$ and $\mathrm{Ti} / \mathrm{Al} / \mathrm{Ni} / \mathrm{Au}(100 \AA / 2000 \AA / 200 \AA / 3000 \AA)$ n-contacts were subsequently deposited. A thick Ti/Au $(200 \AA / 5000 \AA)$ p-pad was then deposited on a portion of the p-contact to be used for probing during test. The simulations are for a $300 \mu \mathrm{m} \times 300 \mu \mathrm{m}$ top-emitting LED with a U-shaped n-contact as shown schematically in Fig. 1. The vertical layer structure is given in Fig. 2 and in Tab. 1. It included an active region of 4 quantum wells that were capped with a p- $\mathrm{Al}_{0.3} \mathrm{Ga}_{0.7} \mathrm{~N}$ blocking layer.

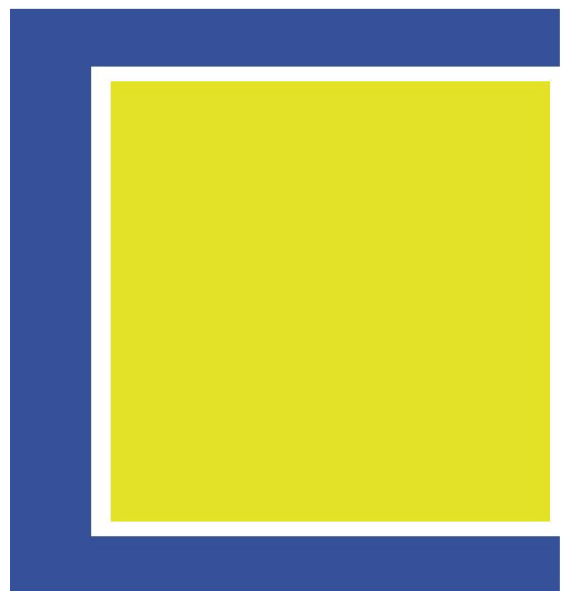

Fig. 1: Schematic top view of LED.

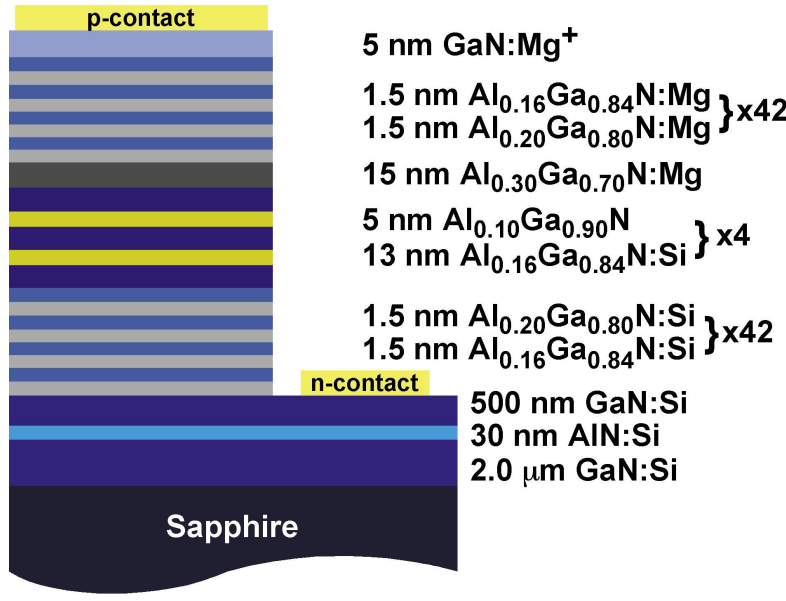

Fig. 2: Vertical structure of LED.

\begin{tabular}{|l|r|l|c|c|}
\hline Material & $\begin{array}{r}\mathbf{d} \\
{[\mathbf{n m}]}\end{array}$ & $\begin{array}{l}\mathbf{n}, \mathbf{p} \\
{\left[\mathbf{1 0}^{\mathbf{1 8}} \mathbf{c m}^{-\mathbf{3}}\right]}\end{array}$ & $\begin{array}{c}\boldsymbol{\tau}_{\text {SRH }} \\
{[\mathbf{n s}]}\end{array}$ & $\mathbf{n}_{\mathbf{r}}$ \\
\hline $\mathrm{p}-\mathrm{GaN}$ & 5 & 1.0 & 1 & 2.77 \\
\hline $\mathrm{p}-\mathrm{AlGaN} \mathrm{SL}$ & 126 & 0.4 & 1 & 2.48 \\
\hline $\mathrm{p}-\mathrm{Al}_{0.3} \mathrm{Ga}_{0.7} \mathrm{~N}$ & 15 & 0.1 & 1 & 2.02 \\
\hline $\mathrm{i}-\mathrm{Al}_{0.1} \mathrm{Ga}_{0.9} \mathrm{~N}$ & 5 & 0.0 & 5 & 2.79 \\
\hline $\mathrm{n}-\mathrm{Al}_{0.16} \mathrm{Ga}_{0.84} \mathrm{~N}$ & 13 & 2.0 & 1 & 2.48 \\
\hline $\mathrm{i}-\mathrm{Al}_{0.1} \mathrm{Ga}_{0.9} \mathrm{~N}$ & 5 & 0.0 & 5 & 2.79 \\
\hline $\mathrm{n}-\mathrm{Al}_{0.16} \mathrm{Ga}_{0.84} \mathrm{~N}$ & 13 & 2.0 & 1 & 2.48 \\
\hline $\mathrm{i}-\mathrm{Al}_{0.1} \mathrm{Ga}_{0.9} \mathrm{~N}$ & 5 & 0.0 & 5 & 2.79 \\
\hline $\mathrm{n}-\mathrm{Al}_{0.16} \mathrm{Ga}_{0.84} \mathrm{~N}$ & 13 & 2.0 & 1 & 2.48 \\
\hline $\mathrm{i}-\mathrm{Al}_{0.1} \mathrm{Ga}_{0.9} \mathrm{~N}$ & 5 & 0.0 & 5 & 2.78 \\
\hline $\mathrm{n}-\mathrm{Al}_{0.16} \mathrm{Ga}_{0.84} \mathrm{~N}$ & 13 & 2.0 & 1 & 2.48 \\
\hline $\mathrm{n}-\mathrm{AlGaN} \mathrm{SL}^{\mathrm{SaN}}$ & 126 & 2.0 & 1 & 2.48 \\
\hline $\mathrm{n}-\mathrm{GaN}^{500}$ & 2.0 & 1 & 2.77 \\
\hline
\end{tabular}

Tab. 1: Epitaxial layer structure with material parameters as used in the simulation (d- thickness, $\mathrm{n}, \mathrm{p}$ - carrier concentration from doping, $\tau_{\mathrm{SRH}}-$ defect recombination lifetime, $\mathrm{n}_{\mathrm{r}}-$ refractive index, $\mathrm{SL}$ - superlattice). 
Figure 3 shows the measured emission spectrum for different injection currents. The peak emission was at $338 \mathrm{~nm}$ and remained fairly constant with increasing current. This indicated very little self-heating of the LED during continuouswave $(\mathrm{CW})$ operation although red-shifting of the emission wavelength from heating is expected to be partially compensated for by blue-shifting of the emission wavelength with increased forward bias. Figure 4 shows bias and top emission power measured with a large-area detector $\left(100 \mathrm{~mm}^{2}\right)$ fixed $\sim 6 \mathrm{~mm}$ above the semi-transparent top p-contact. The maximum light power of $116 \mu \mathrm{W}$ measured at $100 \mathrm{~mA}$ current translates into an external quantum efficiency of $\eta_{\text {ext }}$ $=0.032 \%$. Together with the relatively high voltage of $6.65 \mathrm{~V}$, the wall-plug efficiency becomes $\eta_{\mathrm{wp}}=0.017 \%$. However, the LI curve is non-linear and the highest efficiencies of $\eta_{\mathrm{ext}}=0.037 \%$ and $\eta_{\mathrm{wp}}=0.034 \%$ occur at about $8 \mathrm{~mA}$ injection current. It is estimated that the detector collects about $22 \%$ of the total power emitted by the LED if uniform emission in all directions is assumed. Thus, the total LED efficiency may be significantly higher.

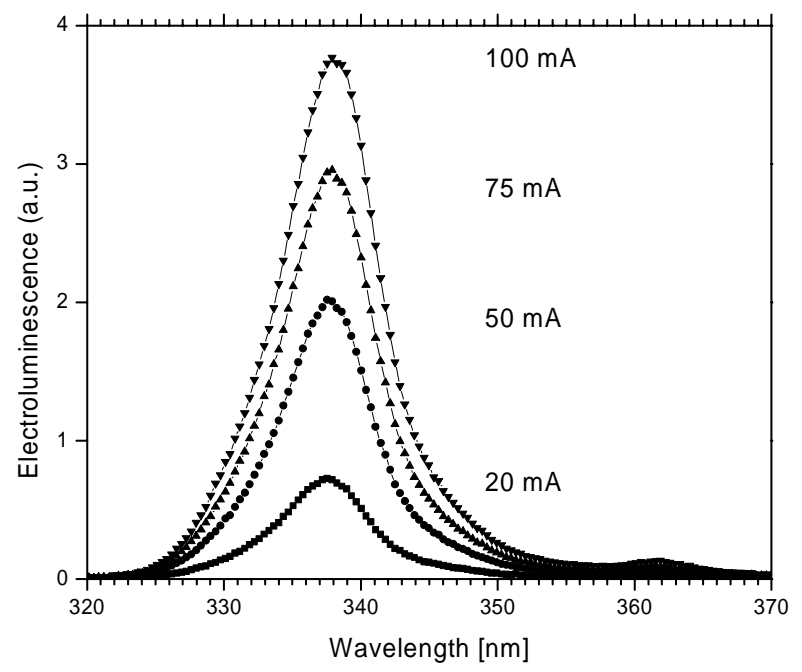

Fig. 3: Electroluminescence measured at different currents.

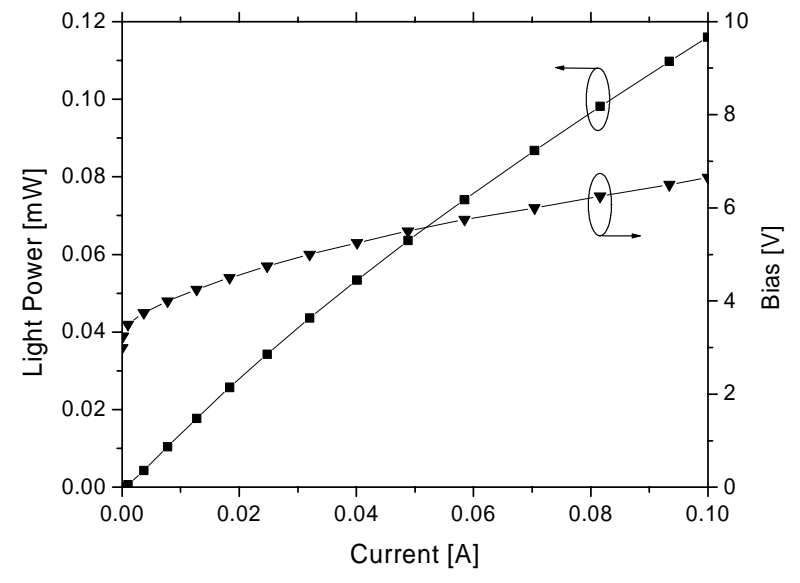

Fig. 4: Measured light-current (LI) and current-voltage (IV) characteristics.

\section{THEORETICAL MODEL}

To simulate nano-scale effects in the AlGaN quantum well and their impact on the device performance we employed the simulation software APSYS. ${ }^{2}$ APSYS self-consistently combined the 3D simulation of carrier transport, quantum well effects, and optical ray tracing. The transport model included drift and diffusion of electrons and holes, Fermi statistics, built-in polarization and thermionic emission at hetero-interfaces, as well as spontaneous and defect related Shockley-Read-Hall (SRH) recombination of carriers. For the quantum wells, Schrödinger and Poisson equations were solved iteratively to account for the quantum well deformation with changing device bias. Spontaneous emission of photons by electron-hole recombination within the quantum well was calculated using a free-carrier model considering the detailed wurtzite valence band structure. Further details of the model can be found elsewhere. ${ }^{2,3}$

\section{MATERIAL PROPERTIES}

An important issue in any device simulation is the inclusion of correct material parameters such as refractive index, carrier mobility, and carrier recombination coefficients, which are often a function of material composition. Published values sometimes spread over a wide range and it is difficult to select the most appropriate numbers. Unless specifically noted otherwise, we employ material parameters from Ref. ${ }^{3}$ which were previously used to obtain excellent agreement with measured $\mathrm{GaN}$ laser characteristics. ${ }^{4}$ The refractive index model for AlGaN was adopted from Laws et al. ${ }^{5}$ and 
the results are listed in Tab. 1 for $340 \mathrm{~nm}$ wavelength. The model included bowing of the $\mathrm{Al}_{\mathrm{x}} \mathrm{Ga}_{1-\mathrm{x}} \mathrm{N}$ energy band gap at room temperature according to Vurgaftman and Meyer ${ }^{6}$

$\mathrm{E}_{\mathrm{g}}(\mathrm{x})=\mathrm{x}(6.28 \mathrm{eV})+(1-\mathrm{x})(3.42 \mathrm{eV})-\mathrm{x}(1-\mathrm{x})(0.7 \mathrm{eV})$

giving slightly larger band gaps than previously assumed, ${ }^{3,7}$ however, the simulated LED spectrum still peaks at slightly longer wavelengths than measured (see next section). Built-in AlGaN interface charges due to spontaneous and piezoelectric polarization were considered using non-linear formulas from Fiorentini et al. ${ }^{8}$ which were in close agreement with experimental observations. ${ }^{7}$ This resulted in smaller charge densities than some linear interpolation schemes. ${ }^{3}$ The fixed interface charge densities are listed in Tab. 2. The superlattices were replaced in the simulation by a bulk $\mathrm{Al}_{0.16} \mathrm{Ga}_{0.84} \mathrm{~N}$ layer. Figures 5 and 6 show the MQW band diagram and the electrostatic field, respectively, with and without interface polarization charges. Built-in polarization caused a strong deformation of the quantum wells accompanied by a strong internal field. Consequently, electrons and holes were separated within the wells and the spontaneous emission rate was reduced, limiting the LED output power. In addition, the impact of the electron blocking layer is reduced by the polarization (see Fig. 5). That impact also depends on the interface band edge offset, which is a rather uncertain parameter. For the GaN/AlN interface, $0.85 \mathrm{eV}$ valence band offset is extracted from the literature as most reliable result, ${ }^{6}$ which translates into the valence band offset ratio of $\Delta \mathrm{E}_{\mathrm{v}} / \Delta \mathrm{E}_{\mathrm{g}}=0.3$ serving as default parameter in our simulation. However, significant deviations from this number are reported and we compare corresponding simulation results in the next section.

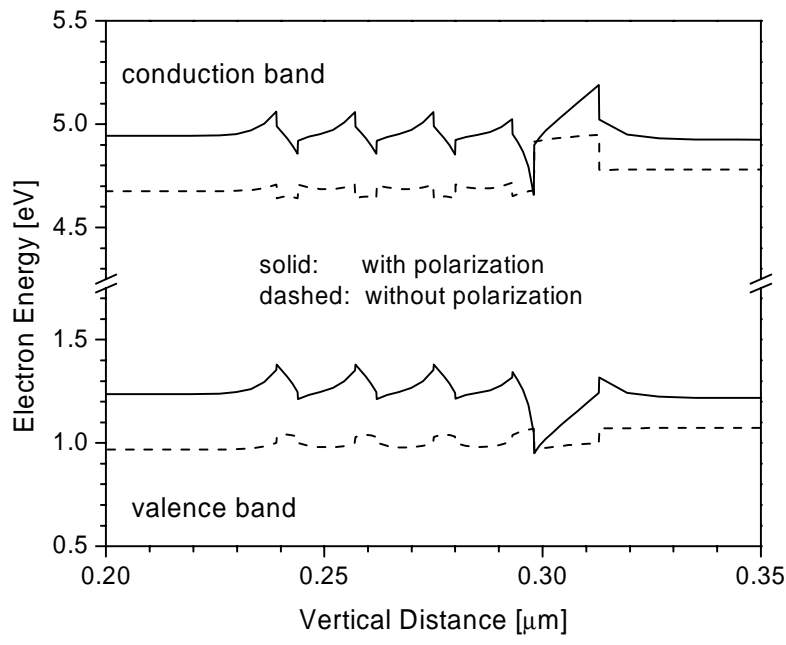

Fig. 5: Energy band diagram with and without built-in interface polarization charges (device center).

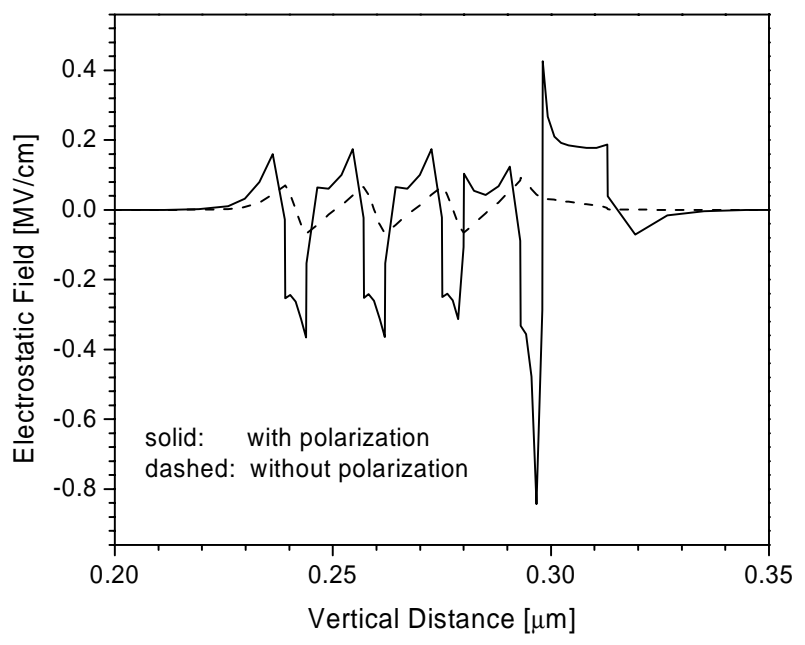

Fig. 6: Vertical electrostatic field with and without built-in interface polarization charges (device center).

\begin{tabular}{|l|l|}
\hline Interface & Polarization Charge \\
\hline $\mathrm{GaN} / \mathrm{Al}_{0.16} \mathrm{Ga}_{0.80} \mathrm{~N}$ & $+6.88 \times 10^{12} \mathrm{~cm}^{-2}$ \\
\hline $\mathrm{Al}_{0.16} \mathrm{Ga}_{0.84} \mathrm{~N} / \mathrm{Al}_{0.10} \mathrm{Ga}_{0.90} \mathrm{~N}$ & $-2.73 \times 10^{12} \mathrm{~cm}^{-2}$ \\
\hline $\mathrm{Al}_{0.10} \mathrm{Ga}_{0.80} \mathrm{~N} / \mathrm{Al}_{0.16} \mathrm{Ga}_{0.84} \mathrm{~N}$ & $+2.73 \times 10^{12} \mathrm{~cm}^{-2}$ \\
\hline $\mathrm{Al}_{0.10} \mathrm{Ga}_{0.80} \mathrm{~N} / \mathrm{Al}_{0.30} \mathrm{Ga}_{0.70} \mathrm{~N}$ & $+9.89 \times 10^{12} \mathrm{~cm}^{-2}$ \\
\hline $\mathrm{Al}_{0.30} \mathrm{Ga}_{0.70} \mathrm{~N} / \mathrm{Al}_{0.16} \mathrm{Ga}_{0.84} \mathrm{~N}$ & $-7.16 \times 10^{12} \mathrm{~cm}^{-2}$ \\
\hline $\mathrm{Al}_{0.16} \mathrm{Ga}_{0.84} \mathrm{~N} / \mathrm{GaN}$ & $-6.88 \times 10^{12} \mathrm{~cm}^{-2}$ \\
\hline
\end{tabular}

Table 2: Fixed polarization charge densities for different types of interfaces in our device. 


\section{SIMULATION AND ANALYSIS}

Figure 7 shows a 3D plot of the LED indicating the recombination rate, which was strongest in the 4 quantum wells. The n-contact was below the region shown and the middle part of the U-shaped metal lies behind the back surface. Enhanced recombination was observed near the left and right edges of the LED, in agreement with experimental observations. This was due to current crowding along the sides with an adjacent n-contact (Fig. 8). The current density was highest in the two corners of the U-shaped contact, about one order magnitude higher than in the center of the LED. These two positions were therefore chosen in the next few graphs to show vertical profiles of different physical properties, all at $100 \mathrm{~mA}$ total injection current.

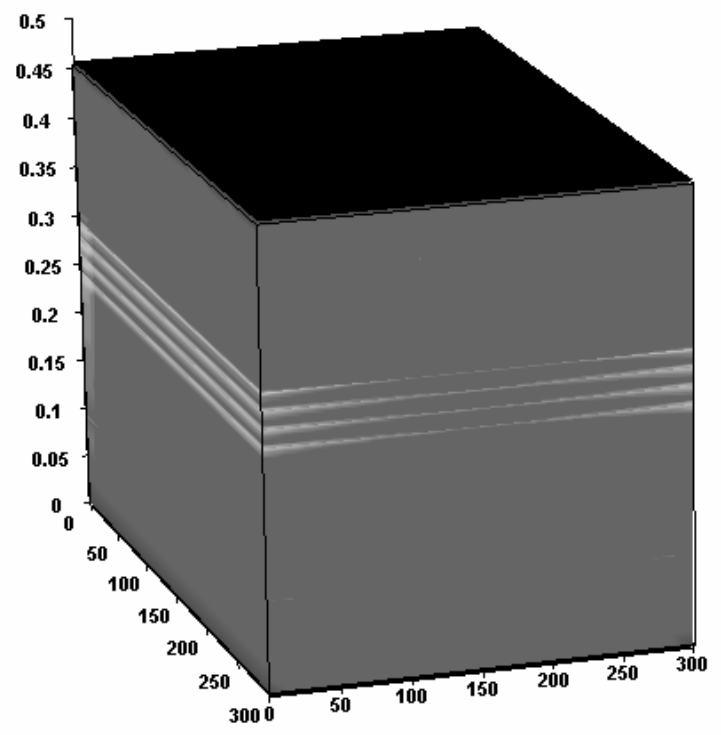

Fig. 7: 3D plot of the LED showing the quantum well recombination rate (the front is equivalent to the right border in Fig. 1).

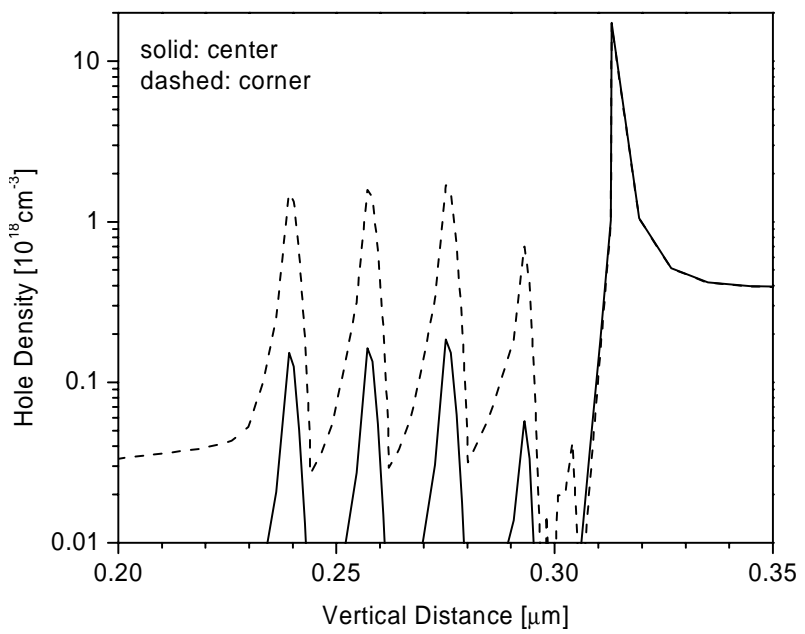

Fig. 9a: Vertical hole density profile for minimum (center) and maximum (corner) current density at $100 \mathrm{~mA}$.

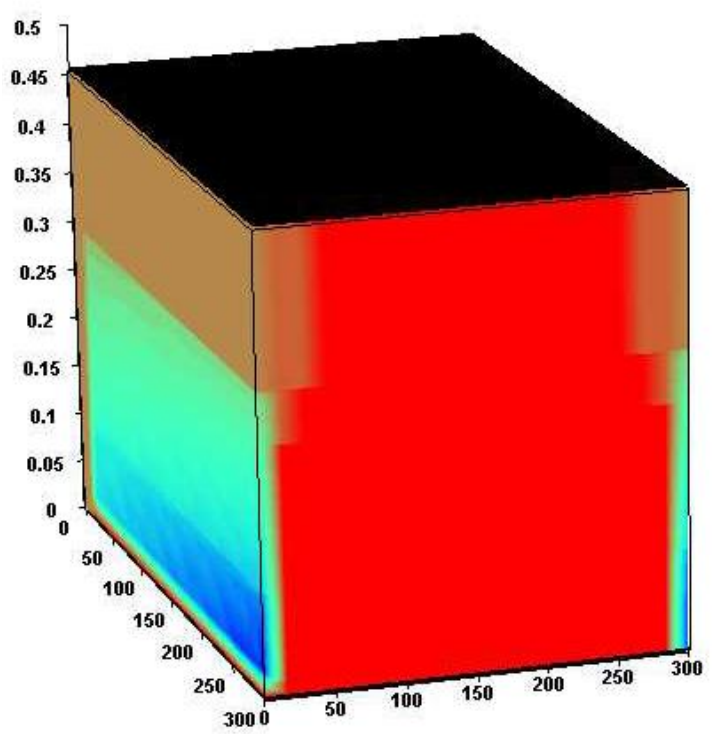

Fig. 8: 3D plot of the LED showing the vertical current density crowding near the side edges.

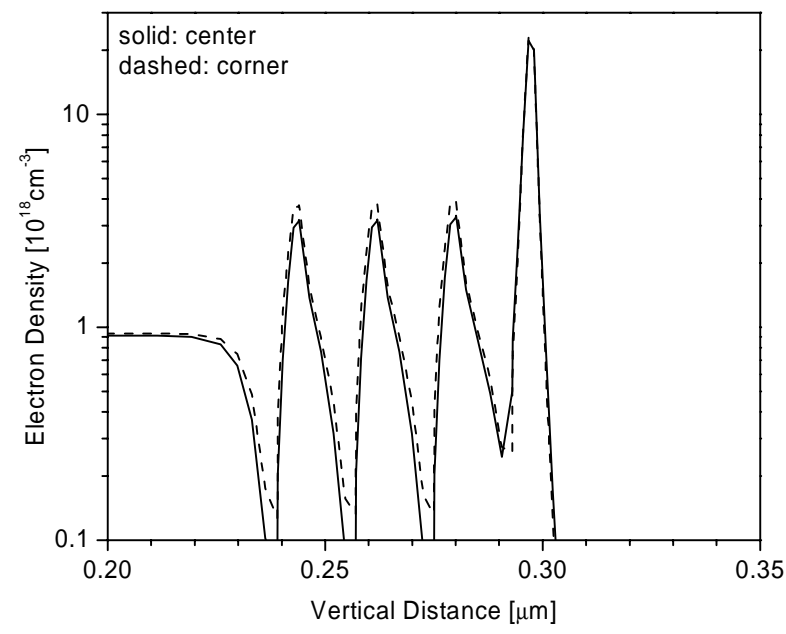

Fig. 9b: Vertical electron density profile for minimum (center) and maximum (corner) current density at $100 \mathrm{~mA}$. 
Figure 9 plots the vertical profile of electron and hole density. Due to the non-uniform hole injection (Fig. 8), the quantum well hole density differed between the two plot positions by almost one order of magnitude. The quantum well electron density was almost the same, due to the much higher electron mobility. The highest hole density occurred above the electron blocking layer due to the valence band edge maximum caused by the negative interface charges (see Fig. 5). Despite the accumulation of holes above the electron blocking layer, the recombination rate was small because the electron density was low at this position.

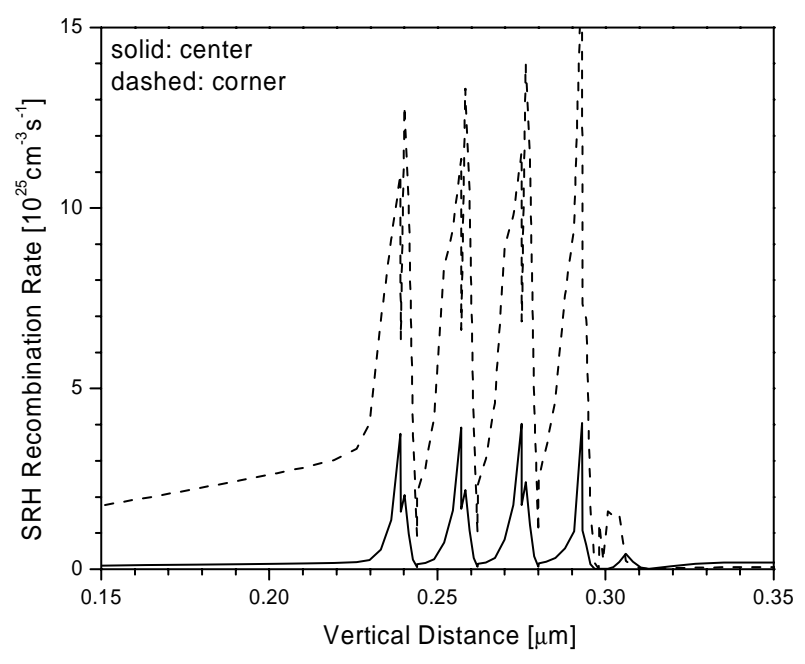

Fig. 10a: Vertical profile of the defect related recombination rate at two positions.

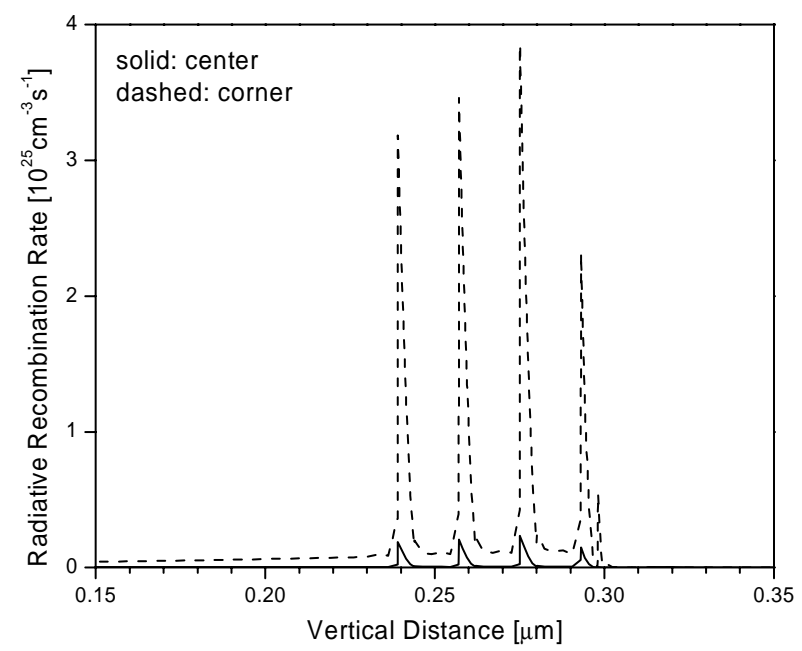

Fig. 10b: Vertical profile of the spontaneous recombination rate at two positions.

Figure 10 shows vertical profiles of the electron-hole recombination rate at both positions. First, Fig. 10a gives the defect-related Shockley-Read-Hall recombination which peaks within the quantum wells, where a non-radiative SRH carrier lifetime of $\tau_{\mathrm{SRH}}=5 \mathrm{~ns}$ is assumed. In fact, this key parameter is unknown for our device and it results from the light vs. current fit below. Other publications estimate several nanoseconds lifetime and it is often assumed that the nonradiative lifetime within the quantum wells is longer than outside the quantum wells (cf. Tab. 1). ${ }^{9}$ The different lifetimes inside and outside the quantum wells generated artificial steps in the curves of Fig. 10a. Due to the strong hole leakage in the corners, significant SRH recombination occured in the n-cladding layer below the MQW. Figure 10b gives the vertical profile of the spontaneous recombination showing much weaker spontaneous recombination compared to the SRH recombination. The strongest photon emission occured in the quantum well corners due to the higher hole density in that region (see Fig. 9a). Some photon generation was also observed in the barrier layers.

Vertical components of the current density are plotted in Fig. 11 (the current is negative because it flows from the top to the bottom). Ideally, electrons and holes meet in the quantum wells and recombine completely. However, Fig. 11a shows that some holes leave the MQW region and enter the lower $\mathrm{n}$-cladding where they recombine with electrons. The hole leakage current was strongest in the corners corresponding to the much stronger total current density and the higher hole concentration in the quantum wells (cf. Fig. 9a). Relatively speaking, about 50\% of the holes leak in the corner while $33 \%$ of the holes leak in the center. Figure $11 \mathrm{~b}$ shows profiles of the vertical electron current. In the corners, some electrons manage to jump over the blocking layer towards the p-contact. This unexpected penetration of the electron blocking layer was supported by the strong deformation of the conduction band edge due to built-in polarization (Fig. 5). The electron quantum level in the top quantum well was lifted up and the effective energy barrier was reduced. In the middle between the contacts, the electron leakage was the same (solid line in Fig. 11b) since the electron density in the top quantum well was almost unchanged (Fig. 9b). However, the vertical electron injection into the MQW from the $\mathrm{n}$-side was even lower than the vertical leakage, due to the strong lateral movement of electrons in the $\mathrm{n}$-cladding. 


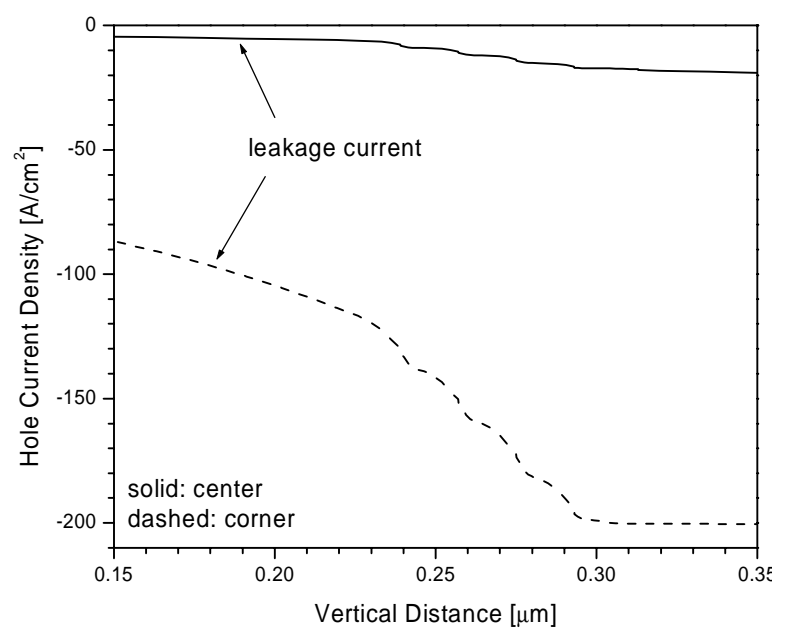

Fig. 11a: Vertical component of the hole current density at two positions.

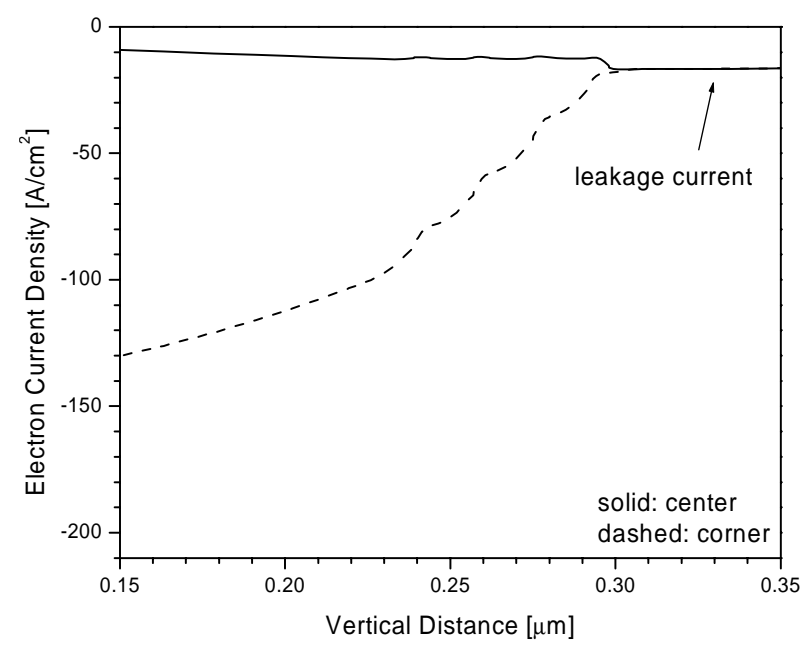

Fig. 11b: Vertical component of the electron current density at two positions.

The above simulations were based on the assumption that at each interface, $30 \%$ of the band gap difference are attributed to the valence band and $70 \%$ to the conduction band. Including some small variations due to strain, the net valence band offset was only $30 \mathrm{meV}$ for the quantum wells, which contributed to the strong hole leakage observed in Fig. 11a. In order to investigate the impact of the band gap offset, we simulated the same device using an offset ratio of $50 \%$ as shown by the dashed line in Fig. 12. The quantum well valence band offset is increased to 46 meV which causes a significant reduction of the hole leakage (solid line in Fig. 13). In fact, the electron leakage from the top quantum well across the blocking layer is now stronger than the hole leakage (dashed line in Fig. 13). This is caused by the reduced $\mathrm{Al}_{0.1} \mathrm{Ga}_{0.9} \mathrm{~N} / \mathrm{Al}_{0.3} \mathrm{Ga}_{0.7} \mathrm{~N}$ conduction band offset, from $243 \mathrm{meV}$ for the default case to $176 \mathrm{meV}$ for $50 \%$ offset ratio. Experimental results indicate a domination of electron leakage in our device, however, the exact band offset remains unknown.

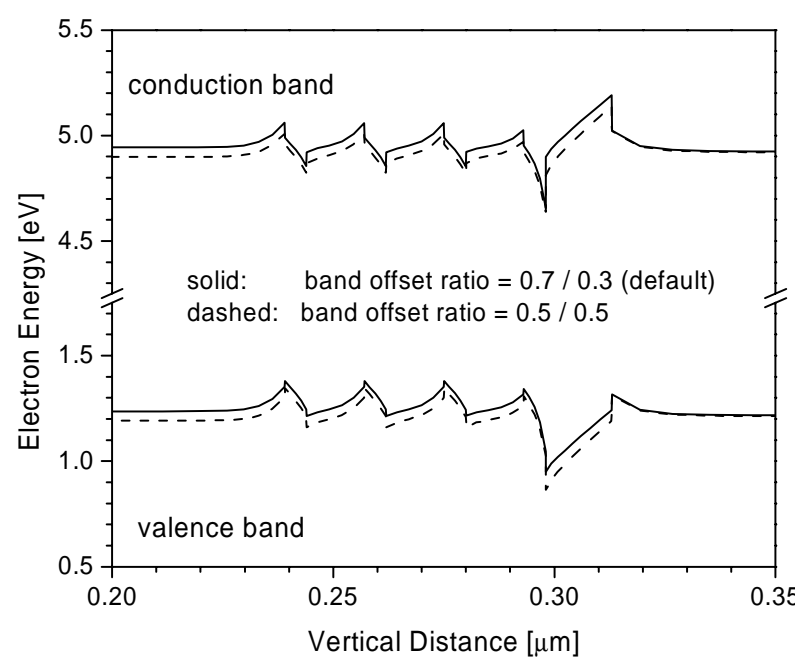

Fig. 12: Energy band diagrams with different offset ratios.

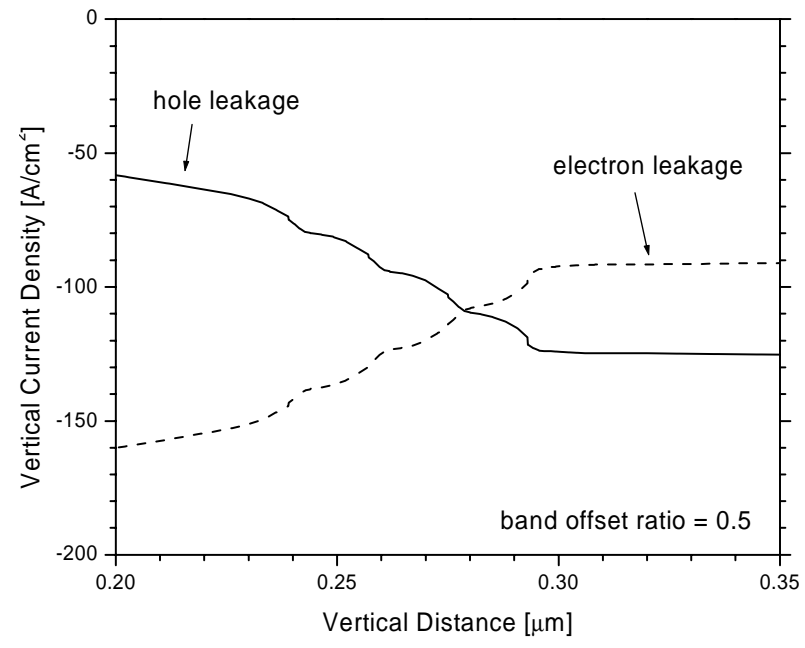

Fig. 13: Vertical current density in the LED corner for a band offset ratio of 0.5 (solid: holes, dashed: electrons). 
The calculated internal emission spectrum is given in Fig. 14, as integrated over the entire LED at different injection currents. Doubling the current resulted in approximately double the emission with hardly any shift of the peak wavelength, similar to the experimental observation in Fig. 3. Despite this agreement, the calculated and experimentally measured line shape differed. Two peaks were distinguishable in the calculation, a main peak at $340 \mathrm{~nm}$ and a slightly weaker one at $342 \mathrm{~nm}$, while the measurement showed only one peak at $338 \mathrm{~nm}$. The calculated longwavelength peak at $342 \mathrm{~nm}$ was attributed to the ground state transition within the top quantum well which was strongly deformed by the built-in polarization and exhibited the lowest possible transition energy in the simulation (see Fig. 5). The peak at $340 \mathrm{~nm}$ was attributed to the higher transition energy in the other three quantum wells that are less deformed by the built-in polarization. The slight difference between the measured and simulated ground state transition could be explained by inaccuracies in Eq. (1) or deviations of the fabricated device from the intended QW composition or shape. The measurement does not, however, show a second peak associated with the top quantum well. There may be less polarization charges in the real device or the shape of the quantum well may deviate otherwise from the theoretical assumption. To further explore this issue, Fig. 15 gives the spectra calculated without polarization charges. The non-symmetric top quantum well had a slightly higher ground state transition energy and produced a small peak at $337 \mathrm{~nm}$ compared to a lower energy ground state transition with polarization included. This indicated that slightly overestimating the polarization charge could explain the discrepancy between the measured and simulated spectrum. The primary peak calculated without polarization at $340 \mathrm{~nm}$ was attributed to the other 3 quantum wells and had approximately the same wavelength as that calculated with polarization. This indicated that the built-in polarization did not cause a significant blue shift of the emission wavelength for quantum wells with polarization and flat symmetric quantum wells (cf. Fig. 5). The polarization does, however, cause a significant separation of electrons and holes, resulting in a significantly reduced peak emission intensity (note the different vertical scales in Figs. 14 and 15). Integration over the entire emission spectrum gives the total internal light power.

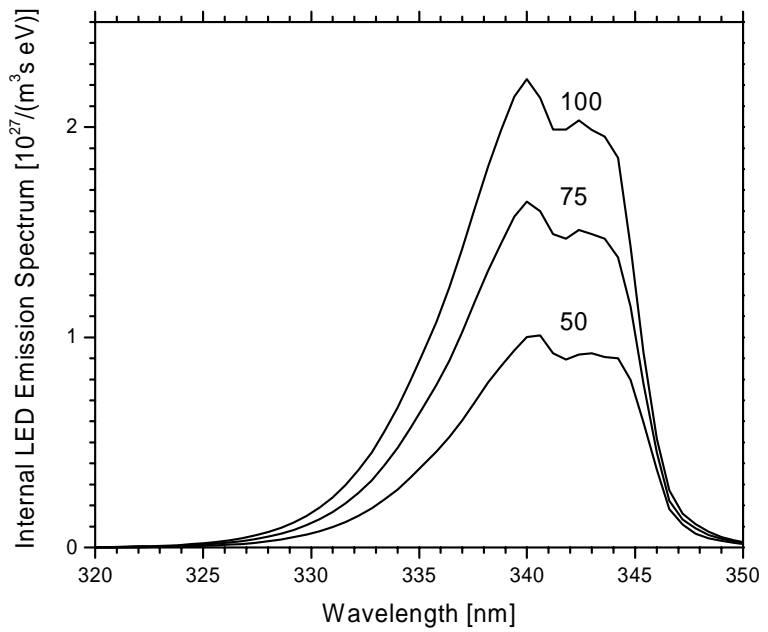

Fig. 14: Internal photon emission spectra with the injection current given as parameter [mA].

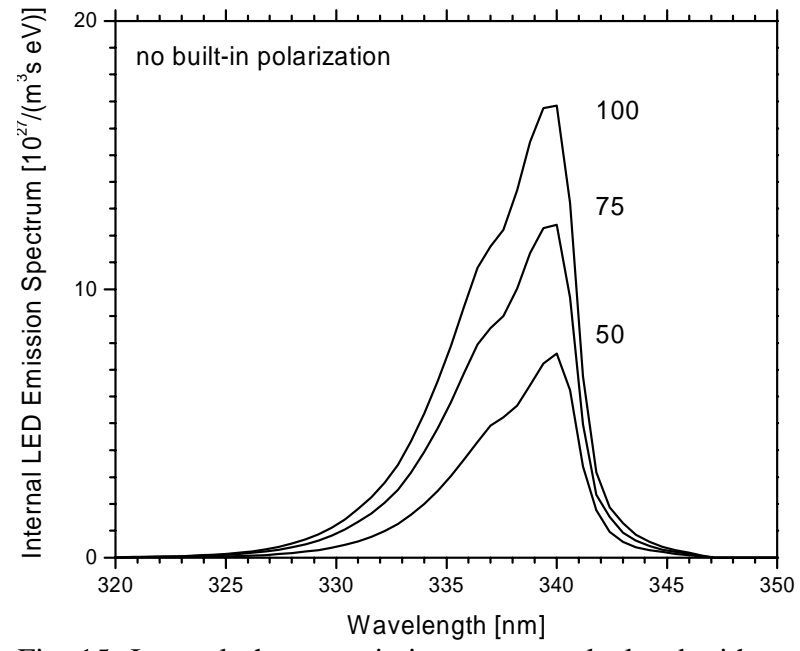

Fig. 15: Internal photon emission spectra calculated without built-in polarization (injection current given as parameter $[\mathrm{mA}])$.

The simulation of the external emission power required 3D ray tracing from every emission point inside the device. Most photons do not escape from the LED, due to total internal reflection, mainly at the device surfaces, and to photon absorption, primarily in the thick GaN layers with a band-to-band absorption coefficient of $\alpha=11 \times 10^{4} \mathrm{~cm}^{-1}$ at 340 $\mathrm{nm}$. Additional absorption occurs in the top transparent contact which comprises a $3 \mathrm{~nm}$ thick Palladium layer (index $1.1, \alpha=18 \times 10^{4} \mathrm{~cm}^{-1}$ ) and a $5 \mathrm{~nm}$ thick Gold layer (index 1.4, $\alpha=59 \times 10^{4} \mathrm{~cm}^{-1}$ ) on top. The detected external quantum efficiency $\eta_{\text {det }}$ gives the number of photons captured by the detector per 100 electron-hole pairs injected into the LED. It is calculated from

$\eta_{\text {det }}=\eta_{\text {int }} \times \eta_{\text {opt }} \times \eta_{\text {cap }}$ 
with the internal quantum efficiency $\eta_{\text {int }}$ (number of photons generated inside the LED per 100 electron-hole pairs injected), the photon extraction efficiency $\eta_{\text {opt }}$ (number of escaped photons per 100 photons generated inside the LED), and the detector capture efficiency $\eta_{\text {cap }}$ (number of detected photons per 100 escaped photons). The last number was not exactly determined in this study. We assumed a best case scenario that all photons that escaped from the LED in an upward direction were detected. For $100 \mathrm{~mA}$ injection current, the above numbers are listed in Table 3 and compared for four cases. The default case (built-in polarization, $\tau_{\mathrm{SRH}}=5 \mathrm{~ns}$, offset ratio $0.7 / 0.3$ ) was closest to the measured external quantum efficiency of $0.032 \%$. With $1 \mathrm{~ns}$ lifetime, more electron-hole pairs were consumed by defect-related recombination, lowering the internal efficiency $\left(\tau_{\mathrm{SRH}}=1 \mu \mathrm{s}\right.$ increases the internal efficiency only to about $1 \%$, i.e., carrier leakage is mainly responsible for the low efficiency simulated). The optical efficiencies were also slightly lower due to a change in the spatial distribution of photon emission inside the LED. Without built-in polarization, the photon generation rate was much stronger (cf. Figs. $14 \& 15$ ) and the internal efficiency was much higher, leading to a significant enhancement of the detected light power. With 50\% band offset ratio, the internal efficiency is strongly reduced since the leaking electrons reduce the effective hole injection into the quantum wells (see Fig. 13).

\begin{tabular}{|l|l|l|l|c|}
\hline Case studied & $\eta_{\text {int }}$ & $\eta_{\text {opt }}$ & $\eta_{\text {cap }}$ & $\eta_{\text {det }}$ \\
\hline default & $0.89 \%$ & $5.2 \%$ & $85 \%$ & $0.039 \%$ \\
\hline$\tau_{\mathrm{SRH}}=1 \mathrm{~ns}$ & $0.54 \%$ & $4.1 \%$ & $81 \%$ & $0.018 \%$ \\
\hline no polarization & $4.40 \%$ & $4.5 \%$ & $94 \%$ & $0.186 \%$ \\
\hline offset ratio $=0.5 / 0.5$ & $0.27 \%$ & $5.9 \%$ & $73 \%$ & $0.012 \%$ \\
\hline
\end{tabular}

Table 3: Calculated quantum efficiencies for the 4 cases simulated.

For all four cases, Fig. 16 plots the detected light power as function of injection current. The slope of the calculated curves was almost constant while the slope of the measured characteristic decreased slightly at higher current. This may be due to slight self-heating, which was excluded from the simulation based on the negligible red-shift of the measured emission peak in Fig. 3. Otherwise, the good agreement between simulation and measurement confirmed the accuracy of our model. The solid line in Fig. 16 was fitted to the measurement by adjusting the SRH carrier lifetime in the quantum well to $5 \mathrm{~ns}$. No literature reports on $\mathrm{AlGaN}$ quantum wells could be found to validate this number, however, the non-radiative lifetime reported for other nitride materials is typically on the order of a few nanoseconds. ${ }^{9}$ However, an overestimation of the photon capture efficiency $\eta_{\text {cap }}$ would lead to an underestimation of the carrier lifetime. In addition, significant uncertainties remain regarding the band offset ratio as well as the actual strength of the built-in polarization, as discussed above.

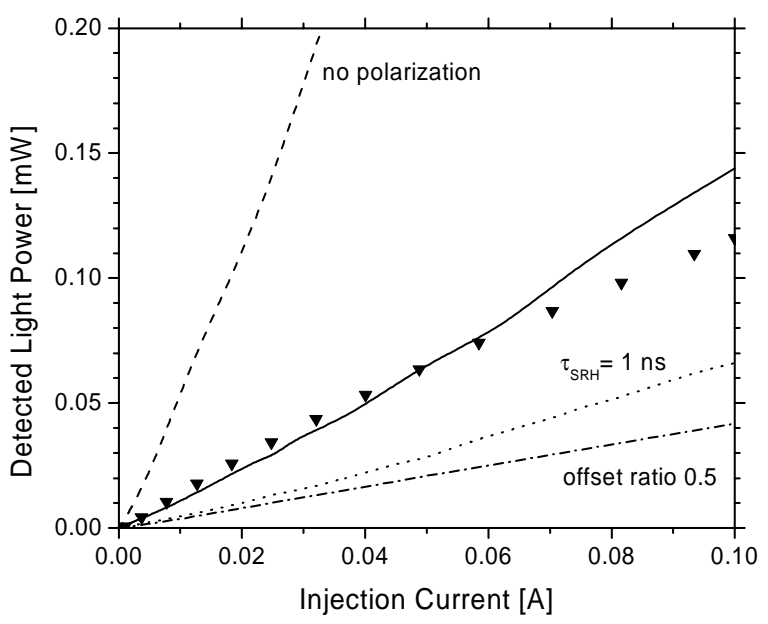

Fig. 16: Calculated power vs. current characteristics for the four cases in Table 3 . The solid line gives the default simulation and the triangles give the measured curve.

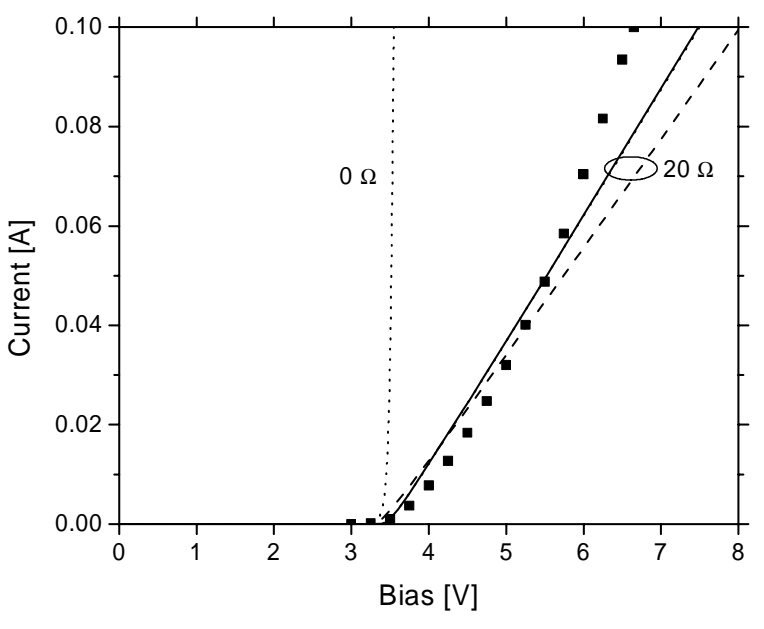

Fig. 17: Calculated current vs. voltage characteristics with (solid, dotted) and without (dashed) polarization (contact resistance given as parameter). The squares give the measured curve. 
Figure 17 compares the calculated current - voltage (IV) characteristics to the measurement. Initially, the calculated IV curve showed a steep turn-on at $3.4 \mathrm{~V}$. In agreement with experimental findings, we added an ohmic top p-contact resistance to the simulation and fitted its value of $20 \mathrm{Ohms}$ to the measurement. This resulted in a linear IV slope that slightly deviated from the superlinear experimental curve. The superlinearity was possibly caused by a Schottky-type contact.

\section{SUMMARY}

The internal device physics of $340 \mathrm{~nm}$ AlGaN/GaN LEDs were analyzed by advanced three-dimensional simulation. The calculated device characteristics showed relatively good agreement with measurements. For the first time, this simulation accounted for the low quantum efficiency measured and gave detailed insight into the underlying mechanisms. The low internal efficiency was mainly caused by carrier leakage from the MQW active region. The photon extraction efficiency was limited by internal absorption, mainly within the $\mathrm{n}$-side GaN layers. The simulations demonstrated the strong impact of nanoscale physics, such as the effect of built-in polarization on the quantum wells, on the measured device performance.

\section{ACKNOWLEDGEMENT}

Support by the Solid-State Lighting and Display Center and by the SUVOS program (John Carrano) is gratefully acknowledged.

\section{REFERENCES}

1 T. Katona, Ph.D. Thesis, "Development of Ultraviolet Nitride-based Light-Emitting Diodes," University of California at Santa Barbara, Electrical and Computer Engineering Department, 2003.

2 APSYS 2003.11.01, Crosslight Software, Burnaby, Canada (http://www.crosslight.com).

3 J. Piprek, Semiconductor Optoelectronic Devices - Introduction to Physics and Simulation, Academic Press, San Diego, 2003.

4 J. Piprek and S. Nakamura, "Physics of High-Power InGaN/GaN Lasers," IEE Proceedings, Optoelectronics, vol. 149, No. 4, pp. 145-151, 2002.

5 G. M. Laws, E. C. Larkins, I. Harrison, C. Molloy, and D. Somerford, "Improved refractive index formulas for the AlGaN and In GaN alloy, “ J. Applied Physics, vol. 89, pp. 1108-1115, 2001.

6 I. Vurgaftman and J. R. Meyer, ” Band parameters for nitrogen-containing semiconductors,” J. Appl. Phys., vol. 94, pp. 3675-3691, 2003.

7 S. Heikman, S. Keller, Y. Wu, J. S. Speck, S. P. DenBaars, and U. K. Mishra, "Polarization effects in AlGaN/GaN and GaN/AlGaN/GaN heterostructures,” J. Appl. Phys., vol. 93, pp. 10114-10118, 2003.

8 V. Fiorentini, F. Bernardini, and O. Ambacher, "Evidence for nonlinear macroscopic polarization in III-V nitride alloy heterostructures," Appl. Phys. Lett., vol. 80, pp. 1204-1206, 2002.

9 S. Nakamura and S. F. Chichibu, Introduction to Nitride Semiconductor Blue Lasers and Light-Emitting Diodes, Taylor \& Francis, London, 2000. 